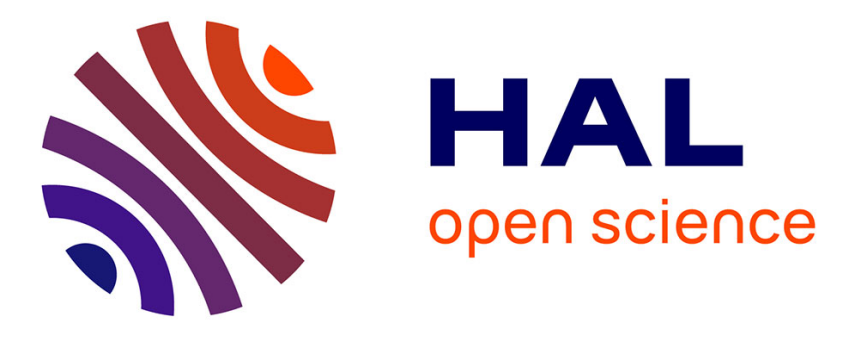

\title{
A data-driven digital-twin prognostics method for proton exchange membrane fuel cell remaining useful life prediction
}

Safa Meraghni, Labib Terrissa, Meiling Yue, Jian Ma, Samir Jemei, Noureddine Zerhouni

\section{To cite this version:}

Safa Meraghni, Labib Terrissa, Meiling Yue, Jian Ma, Samir Jemei, et al.. A data-driven digital-twin prognostics method for proton exchange membrane fuel cell remaining useful life prediction. International Journal of Hydrogen Energy, 2020, 46 (2), pp.2555-2564. 10.1016/j.ijhydene.2020.10.108 . hal-03186600

\section{HAL Id: hal-03186600 \\ https://hal.science/hal-03186600}

Submitted on 5 Oct 2021

HAL is a multi-disciplinary open access archive for the deposit and dissemination of scientific research documents, whether they are published or not. The documents may come from teaching and research institutions in France or abroad, or from public or private research centers.
L'archive ouverte pluridisciplinaire HAL, est destinée au dépôt et à la diffusion de documents scientifiques de niveau recherche, publiés ou non, émanant des établissements d'enseignement et de recherche français ou étrangers, des laboratoires publics ou privés.

\section{(c)(1)}

Distributed under a Creative Commons Attribution| 4.0 International License 


\title{
A data-driven digital-twin prognostics method for proton exchange membrane fuel cell remaining useful life prediction
}

\author{
Safa Meraghni ${ }^{a, b}$, Labib Sadek Terrissa $^{a}$, Meiling Yue ${ }^{b, c, *}$, Jian $\mathrm{Ma}^{\text {d,e }}$, \\ Samir Jemei ${ }^{b, c}$, Noureddine Zerhouni ${ }^{b, c}$ \\ a LINFI Laboratory, University of Biskra, Algeria \\ ${ }^{\mathrm{b}}$ FEMTO-ST Institute (UMR CNRS 6174), Univ. Bourgogne Franche-Comte, France \\ ${ }^{c}$ FCLAB Research Federation (FR CNRS 3539), Univ. Bourgogne Franche-Comte, France \\ d School of Reliability and Systems Engineering, Beihang University, China \\ e National Key Laboratory of Science and Technology on Reliability and Environmental Engineering, China
}

\begin{abstract}
Prognostics and health management of proton exchange membrane fuel cell (PEMFC) systems have driven increasing research attention in recent years as the durability of PEMFC stack remains as a technical barrier for its large-scale commercialization. To monitor the health state during PEMFC operation, digital twin (DT), as a smart manufacturing technique, is applied in this paper to establish an ensemble remaining useful life prediction system. A data-driven DT is constructed to integrate the physical knowledge of the system and a deep transfer learning model based on stacked denoising autoencoder is used to update the DT with online measurement. A case study with experimental PEMFC degradation data is presented where the proposed data-driven DT prognostics method has applied and reached a high prediction accuracy. Furthermore, the predicted results are proved to be less affected even with limited measurement data.
\end{abstract}

\footnotetext{
* Corresponding author. FEMTO-ST Institute (UMR CNRS 6174), Univ. Bourgogne Franche-Comte, France.

E-mail address: meiling.yue@femto-st.fr (M. Yue).
} 


$\begin{array}{ll}\text { Nomenclature } \\ \Sigma & \text { standard deviation } \\ \text { AE } & \text { autoencoder } \\ \text { DT } & \text { digital twin } \\ \text { PEMFC } & \text { proton exchange membrane fuel cell } \\ \text { PF } & \text { particle filter } \\ \text { PHM } & \text { prognostics and health management } \\ \text { ReAcc } & \text { relative accuracy } \\ \text { RUL } & \text { remaining useful life } \\ \text { SDA } & \text { stacked denoising autoencoder }\end{array}$

\section{Introduction}

In an era of accelerating change, the imperative to limit climate change and achieve sustainable growth is strengthening the momentum of the global energy transformation. A "hydrogen economy era" is coming into the human's horizon towards establishing a cleaner energy system [1]. In this context, fuel cells are regarded as the technology of choice to maximise the potential benefits of hydrogen in terms of efficiency [2]. Proton exchange membrane fuel cell (PEMFC) is currently the leading technology for light-duty vehicles and materials handling vehicles, and to a less extent for stationary and other applications [3]. However, two primary challenges have limited its massive commercialization, i.e. cost and durability [4]. The unsatisfied durability and reliability of the current PEMFC systems can be associated with the high maintenance cost [5], while non-optimized operation could be a critical reason leading to the unexpected shutdowns and further degradation of the components [6]. Efforts have been made to improve its durability: working on the materials, reducing the causes of degradation, improving the structural design, implementing new supervision and management designs, etc.

Prognostics and health management (PHM), as a newly developed discipline derived originally from condition-based maintenance [7], has been applied to monitor and predict the health state of PEMFC systems [8,9]. Various prognostics approaches have been investigated for PEMFC applications aiming at assessing its health state and predicting its remaining useful life (RUL). For example, in the literature, echo-state networks [10] and adaptive neuro fuzzy inference system [11] have been used as data-driven approaches to predict PEMFC's RUL. Besides, Javed et al. have used a constraint-based summation wavelet-extreme learning machine to learn the system behaviour directly from the data and to train a single-layer feed-forward neural network to predict the RULs of the PEMFC [12]. However, as data-driven prognostics approaches are model-free and they rely only on previously observed data when the measurements are not sufficient, the predicted results can be highly influenced [13]. Although PHM methods and solutions have engaged in RUL prediction, deficiencies are left over due to the lack of connections between physical knowledge and prediction solutions [14]. As a result, online prediction performance has been limited due to the shortage of solutions to collect, connect and control sensor data [15], and preprocess it through efficient numerical models so that the models that are used for RUL prediction are rarely updated over time. Also, physical phenomena and their degradation profiles are not considered, which further limits the PHM deployment at the machine level. To overcome these drawbacks, the concept of a Digital Twin (DT) as a smart manufacturing technique has begun to find its place in different PHM process stages, ranging from health state monitoring, diagnostics and prognostics.

DT is a virtual living model of a physical system that can update its state continuously as the physical system changes. It consists of three parts: physical space, virtual space and the linkage between the physical space and the virtual space [16]. Instead of relying only on the sensor data to predict deviations from the standard operation, DT provides a synthetic way to interpret and integrate the collected data with the expertise knowledge constructed in the virtual space [17]. DT is able to deliver accurate health state predictions of complex systems based on the continuous adaptation to the changes of environment and operation conditions.

The first proposition of applying DT in PHM field is using it to predict the aircraft structural health state [18]. Later on, more interests have been generated. Tao et al. have proposed a method of DT-based PHM to predict the health state of wind turbines using the interaction mechanism and fused data of DT [19]. To monitor a hydraulic support, Xie et al. [20] have proposed a DT through a virtual monitoring method. The attitude of the hydraulic supports is combined with the virtual digital model using an information fusion algorithm. Zakrajsek et al. [21] have proposed a DT for health monitoring of tires at touchdown to estimate the failure probability and improve the mission decision, therefore, save the cost. Towards fault diagnosis, Xu et al. have proposed a DT for car body-side production line [22] and Wang et al. have presented a DT for rotating machinery fault diagnosis [23].

In the energy domain, many works have introduced DT to coordinate and support specific system goals, such as energy management [24,25], energy optimization [26] and monitoring the behaviour of the system, etc [27]. A recent research has proposed a DT that combines multi-physical and data-driven models for the multi-physics prediction of a PEMFC [28]. A dataset is generated in 100 different operating condition scenarios using a 3D multi-physical model of the PEMFC. Next, an artificial neural network and a support vector machine have been applied to predict different physical quantities of a PEMFC using the generated data. To the best of our knowledge, the DT has not been developed for the PEMFC prognostics in the literature. To fill this gap, this paper proposed a data-driven DT based on PEMFC behaviour using a stacked autoencoders model. The health prediction is performed then using transfer learning based on the analysis and mining of similar PEMFC degradation data with individual variations. The main contributions of this paper are as follows:

- A DT structure is extended to the energy domain, which is applied for PEMFC prognostics.

- A data-driven DT model of PEMFC is established on the digital side. 
- The degradation behaviour of the PEMFC is captured by stacked autoencoders, while the online prognostics is performed by transfer learning in the DT framework.

- A case study is presented to evaluate the performance of the proposed method using the real data collected from the long-term PEMFC degradation experiment.

Following parts of this paper are arranged as follows: Section State of the art presents the principles and the state of the art of applying DT in PHM. Our proposed data-driven DT prognostics method for PEMFC is described in Section Proposed data-driven DT prognostics method where a prognostics model based on deep learning neural network is built. A case study of PEMFC RUL prediction based on experimental data is then presented in Section Case study. Finally, Section Conclusion concludes the contribution of this paper.

\section{State of the art}

DT adapted for prognostics purpose can be regarded as a virtual representation of the health state of the studied physical system, which is equipped with sensors. These sensors give operation data of the physical system in real time. On the digital side of DT, the model is built and updated to predict the health state of the physical system and make real-time decisions.

The creation of DT based on physical models is not always straightforward due to the complexity of the machine in modern production plants. To this concern, many works have been interested in developing data-driven DT. For example, Zhuang et al. have focused on managing the complex product assembly shop-floors, in which a framework based on digital twin and big data technologies have been proposed to promotes the realization of predictive manufacturing in the product assembly stage [29]. Tao et al. have proposed a datadriven digital twin to deal with data logging problem in the product life cycle [30]. To reschedule the aircraft mission dynamically based on its structural damage, Kapteyn et al. have proposed a data-driven physics-based DT where the physical model has been used to generate a large dataset containing the predictions of the aircraft structure states. Then, the generated dataset has been used to train the machine learning model, and update the DT state. Min et al. have proposed a machine learning method to update the DT used in petrochemical industry [31]. The collected historical data has been used to train the model and the on-line collected operation data has been used to update the twin dynamically based on the changing environment in real time.

Previous works of data-driven DT for prognostics purpose have been found in various applications, while it has been rarely developed for fuel cell systems. As PEMFC system refers to a PEMFC stack and all its auxiliaries, which is a multi-scale system varying from nano-scale chemical reactions to system-scale interactions, a great number of parameters exist influencing its performance and bringing difficulties in system maintenance [32]. Besides, the health prediction of PEMFCs could be influenced by their operating profiles, as well as the intercell differences [33]. Developing one model for each condition is time-consuming, while an adaptive model seems promising and can ensure the generalizability. Therefore, instead of constructing a physical digital model, a data-driven DT is proposed in this paper for PEMFC prognostics applications. Besides, a transfer learning method is applied to address the problem of limited data and also save the time to construct the intelligent neural network.

To sum up, developing a data-drive DT structure for PEMFC prognostics is able to capture the degradation behaviour of the fuel cell regardless of the operation conditions and other varying physical parameters. The issues of limited learning data and computation burden are addressed by transfer learning. The proposed method is described in the following sections.

\section{Proposed data-driven DT prognostics method}

In this work, we assume that the physical space is composed of the PEMFC and the sensors required to collect the data in real-time. We also assume that the connection between the digital space and the physical space is already established. Thus, we focus on creating a model to mirror the PEMFC behaviors on the digital side.

In this section, a data-driven DT prognostics method is proposed for PEMFC applications, which is able to estimate the health state of the fuel cell and predict its remaining useful life.

\section{Framework of proposed method}

Fig. 1 shows the framework of the proposed data-driven DT prognostics method applying to PEMFCs. The digital space consists of two phases: modeling phase and updating phase. At first, an offline modeling phase is deployed to build the DT model to mirror the behaviour of the PEMFC based on the historical data. This is performed based on transfer learning where the historical data are used to train the model to get the

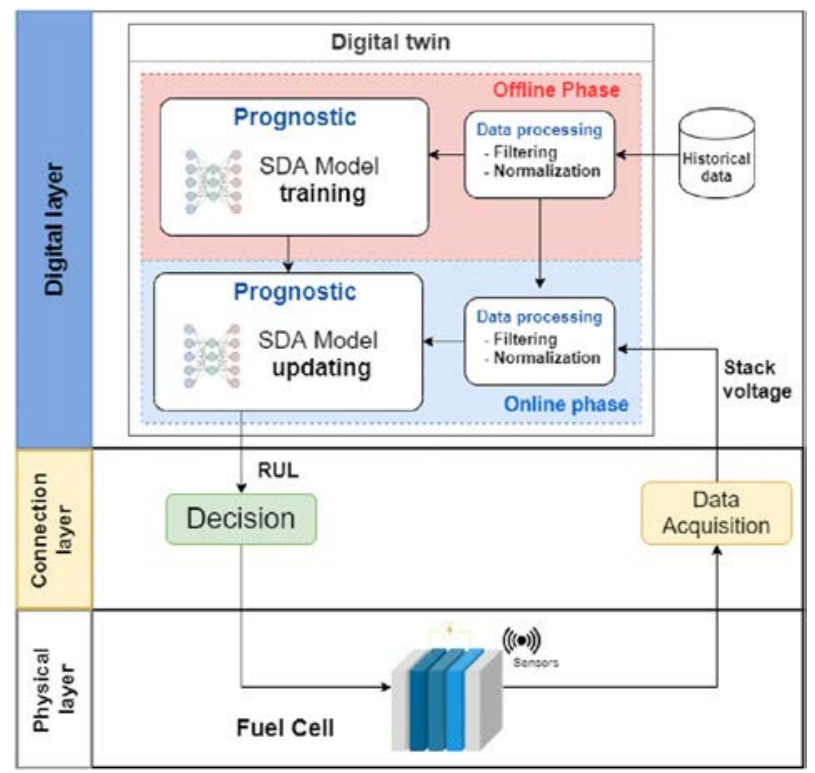

Fig. 1 - Proposed data-driven DT prognostics method. 
PEMFC behaviour model. The input of the model is the sensor data of the plant, which can indicate performance degradation. For PEMFCs, their performance is evaluated on a stack level so that the stack voltage can be selected as the performance indicator as it degrades along with the continuous operation. The output of the model is, therefore, the RUL.

This is the digital side of the prognostics DT, i.e. in virtual space. Then, for the online phase, the physical system, namely, the target fuel cell system, is connected to the digital model in order to transfer the online measurement to the digital side. Based on the real-time data obtained from online operation, the digital model is updated, while the RUL of the target PEMFC is calculated. According to the predicted RUL, maintenance decisions or strategies could be made to avoid system shutdowns owing to the predictive feature.

\section{Prognostics model based on SDA}

Treating PEMFC as a black box, the technique of autoencoder (AE) is used to find the virtual link between its input and output. AE is a specific type of feedforward neural network where the input has the same dimension as the output. It compresses the input into a lower-dimensional code and then reconstruct the output from this representation. It has three layers: an input layer, a hidden (encoding) layer, and a decoding layer and it is trained to learn a function:

$$
h(\widehat{\mathbf{x}}) \approx \mathbf{x}
$$

where the input data $\mathrm{x}$ is firstly mapped into a hidden layer $\mathrm{y}$, parameterized by $\theta=\{\mathrm{W}, \mathrm{b}\}, \mathrm{y}=\mathrm{f}_{\theta}(\mathrm{x})=\mathrm{s}(\mathrm{Wx}+\mathrm{b})$. Then the output $\widehat{\mathbf{x}}$ is reconstructed from $\mathrm{y}, \widehat{\mathbf{x}}=g_{\theta}^{\prime}(\mathbf{y})=\mathrm{s}\left(\mathrm{W}^{\prime} \mathbf{y}+\mathbf{b}^{\prime}\right)$. The parameters are optimized to minimize the average reconstruction error between the input and the output [34]:

$\theta, \theta^{\prime}=\arg \min \frac{1}{n} \sum_{i=1}^{n} L\left(x^{i}, \hat{x}^{i}\right)$

where $L$ is a loss function, for example, the traditional squared error $L(x, \widehat{x})=\|x-\widehat{x}\|^{2}$.

Besides, in order to force the hidden layer to discover more robust features and prevent it from simply learning the identity, the $\mathrm{AE}$ can be trained to reconstruct the input from a corrupted version of it, the so-called denoising AE. This can be done by adding some noise to the input data and make the $\mathrm{AE}$ learn to remove it. By this means, the encoder will extract the most important features and learn a robust representation of the data.

Denoising $\mathrm{AE}$ has been found as a way to pretrain deep neural networks by combining them in a stacked way, i.e. SDA [35]. SDA is constructed by multiple denoising AEs stringing together, in which the output of the layer below is the input to the current layer. The unsupervised pre-training of such an architecture is done one layer at a time. Each layer is trained as a denoising $\mathrm{AE}$ by minimizing the error in reconstructing its input. Once all layers are trained, the network goes through the second stage of training called fine-tuning. To do this, a logistic regression layer is added on the top of the network, then the entire network is trained and only the encoding parts of each AE are considered [36]. The process of training an SDA prognostics model is summarized as:

\section{Pre-training}

A sequence of shallow AEs is trained with unsupervised data, i.e. the stack voltage of PEMFC, based on (1) and (2).

\section{Fine-tuning}

The pre-trained encoders are used for training the last layer, which is the RUL output layer. The weights and biases obtained in the pre-training step are used to initialize the hidden layers of the SDA. Here, labelled supervised data is used to minimize the prediction error, written as:

$\theta^{*}, \theta \prime^{*}=\arg \min \frac{1}{n} \sum_{i=1}^{n} L\left(V_{L}^{i}, R U L_{L}^{i}\right)$

where $V_{L}$ is the labelled stack voltage, $R_{L} L_{L}$ is the labelled RUL values and the loss function $L$ is calculated through $L\left(V_{L}^{i}\right.$, $\left.R U L_{L}^{i}\right)=\left\|V_{L}^{i}-R U L_{L}^{i}\right\|^{2}$. Then, the weights and biases of the entire SDA are updated through a backpropagate algorithm.

\section{Online RUL prediction}

Once the SDA prognostics model is built, the next step is to connect it with the physical space. The process of training the SDA model and updating it using online data is shown in Fig. 2. Four phases including the above described SDA modeling are designed for online RUL prediction, summarized as follows:

\section{Data collecting}

While the historical data is used to build the SDA prognostics model, the measure stack voltage of the target PEMFC from online operation is collected as testing data, which is injected into the trained prognostics model and is used to update the model.

\section{Data pre-processing}

Raw data with too much noise cannot be applied directly for learning. In pre-processing phase, the collected data is first going to be filtered and normalized. In this step, the historical data should be prepared to model the SDA so that it is divided into training datasets and testing datasets with batches for forward and backpropagation learning. As our purpose is to obtain RULs, labels are set to the processed data as the prior knowledge of RULs.

\section{SDA modeling}

SDA architecture is built and parameters are initialized with random values. By injecting historical datasets, learning and backpropagation are gone through until the maximum number of epochs is reached. This same procedure is applied to each layer of the SDA. 


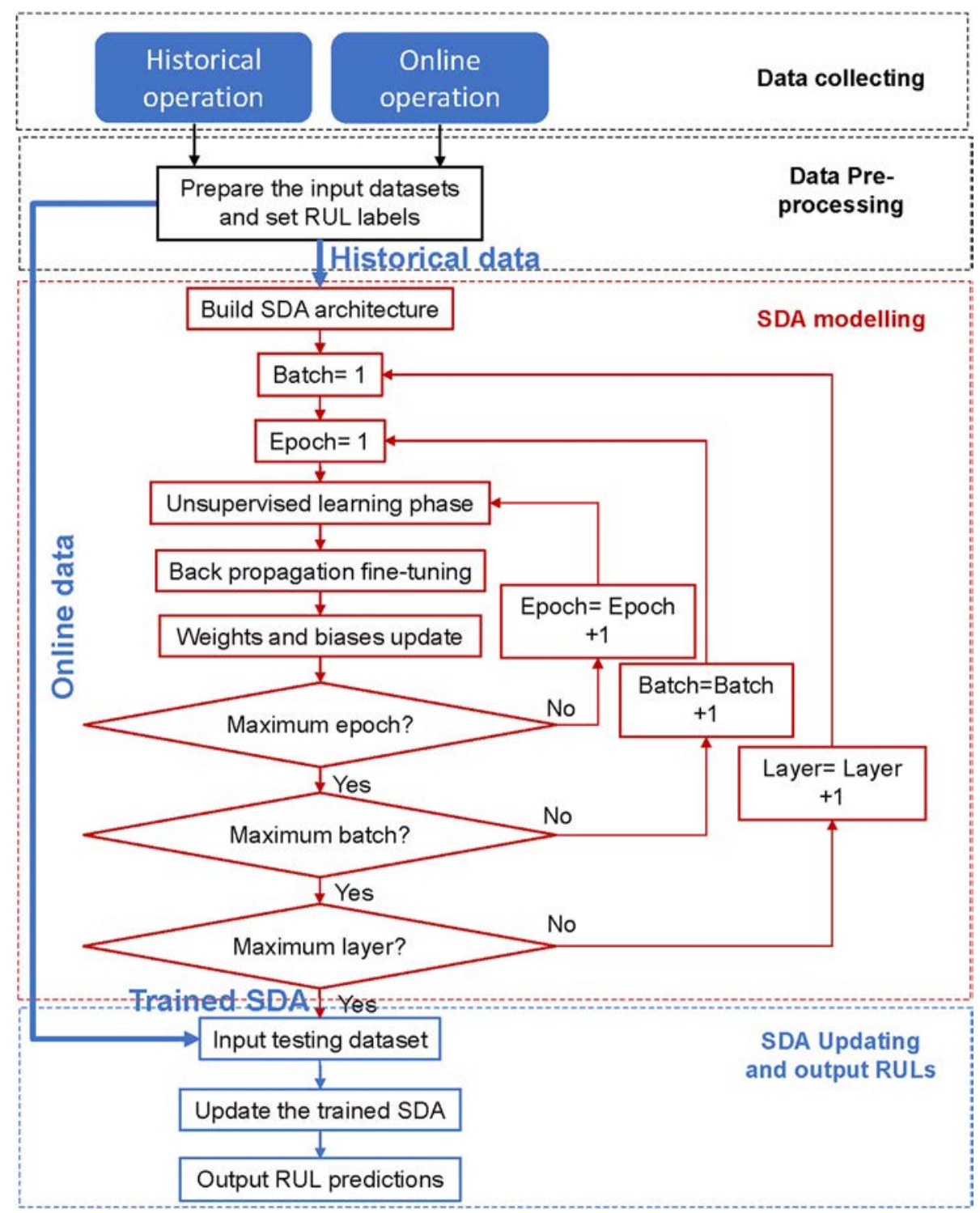

Fig. 2 - DT updating phase: online RUL prediction using the proposed SDA model.

RUL prediction

The online measured stack voltage is then injected to the trained SDA model. The parameters of SDA are updated and the RUL predictions are calculated through the prognostics model.

\section{Case study}

This section presents a case study of PEMFC prognostics using the proposed data-driven DT prognostics method. A set of experimental PEMFC degradation data is used to evaluate the prognostics results.

\section{PEMFC degradation data description}

A PEMFC stack ageing experiment has been launched at FCLAB Research Federation, France (http://eng.fclab.fr/). The tested fuel cell is a 5-cell PEMFC stack with $100 \mathrm{~cm}^{2}$ active area, which is operated under a constant load profile with a current density of $0.6 \mathrm{~A} / \mathrm{cm}^{2}$ [37]. The tested PEMFC has gone through its natural ageing process without any faulty conditions. Characterizations of the stack have been conducted every

Table 1 - PEMFC ageing experiment parameters [37].

\begin{tabular}{ll} 
Parameter & Value \\
\hline Current & $60 \mathrm{~A}$ \\
Anode stoichiometry & 1.5 \\
Cathode stoichiometry & 2 \\
Absolute pressure of input $\mathrm{H}_{2}$ & $150 \mathrm{kPa}$ \\
Absolute pressure of input air & $150 \mathrm{kPa}$ \\
Maximum pressure different & $30 \mathrm{kPa}$ \\
Temperature & $60 \circ \mathrm{C}$ \\
Anode relative humidity & Drying \\
Cathode relative humidity & $60 \%$
\end{tabular}


week, i.e. every $168 \mathrm{~h}$ ideally. Some parameters of the ageing experiment are listed in Table 1.

The ageing experiment has lasted for $1750 \mathrm{~h}$ with average cell voltage degraded $10 \%$, approximately. The five cell voltages of the tested PEMFC stack obtained from the test bench are plotted in Fig. 3. During the experiment, the operation is interrupted by some characterization tests, i.e. polarization curve generation and electrochemical impedance spectroscopy (EIS) application, causing reversible degradation phenomena of the stack. It is indicated by the power recoveries, which may be caused by the major changes in gas flows after the interruptions of resting periods. The reversible degradation is of transient regime and will disappear shortly once the stack comes back to its normal operation [38].

As shown in Fig. 3, the five cells on the same stack are similar, but do not degrade in the same manner due to the location variation. The cells next to the edges of the stack degrade faster than the cells in the middle [39]. Therefore, the five degraded cell voltages are used to verify the effectiveness of the proposed PEMFC prognostics method. Four of the datasets are used as the historical datasets to build the SDA prognostics model, while the other dataset is supposed to be obtained from online operation, which is separated into two parts, one part is injected into the model to update model parameters and the other part is used to evaluate the results.

\section{Data pre-processing}

As it is noted that the peaks observed in the voltage signal are due to the weekly PEMFC characterizations, which are nonsense regarding irreversible stack degradation. However, under the circumstance that there are few samples available for learning (the cell voltage are recorded per hour), the peak data tends to be unpredictable and will have a significant influence on the data-driven prognostics performance. A multiplicative model is used to decompose the observed signal into level, trend, seasonal values and residual values, written as:

$y(t)=$ Level $*$ Trend*Seasonality*Residual

The multiplicative decomposition is suitable for the signals, in which the magnitude of the seasonal fluctuations, or the

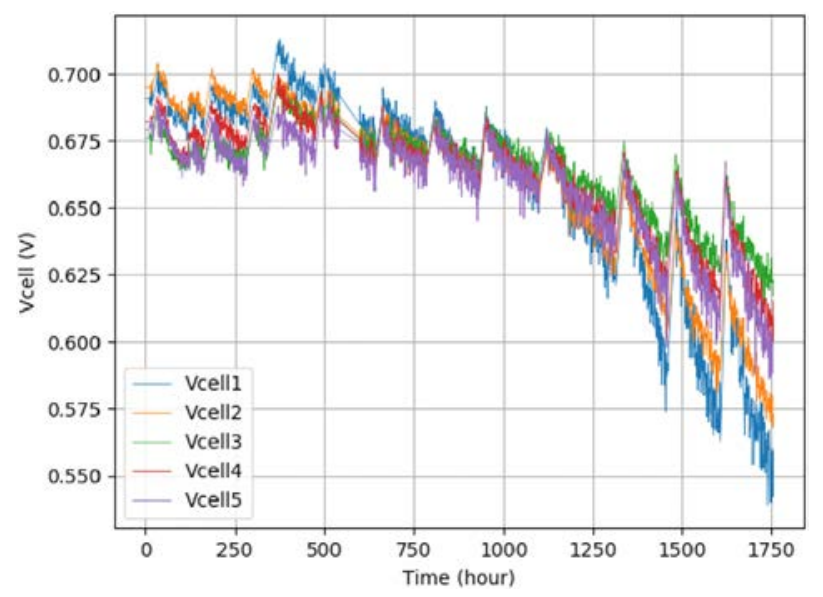

Fig. 3 - Experimental fuel cell voltage. variation around the trend, varies with the level of the time series. As in our case, the fuel cell characterization is performed each week during the long-term degradation experiment and causes different levels of voltage recoveries, the multiplicative model is chosen to deal with the varying time series magnitude. The decomposition result of $V_{\text {cell1 }}$ is plotted in Fig. 4, in which the variable "trend" shows the irreversible degradation trend of the PEMFC, while all the seasonal variations, noise and peaks are decomposed into other terms.

\section{SDA setup}

\section{Key parameters}

After repeated trials, the SDA used for this study is set up with the parameters listed in Table 2. The model input is the labelled decomposed voltage trend and the model output is the labelled RUL values. Two hidden layers are used to recognize the PEMFC voltage degradation feature and a behaviour model of its RUL is built based on the learned features. The transfer function is sigmoid. The batch size is 16 to separate input data into groups and a maximum epoch number is defined as 20 . The sparsity criterion is set as 0.15 .

\section{Drop-out}

Drop-out refers to ignoring some of the neurons during the training phase which is chosen at random. Drop-out technique is commonly used in the training phase of a deep learning network in order to prevent over-fitting of the training data, especially when the training samples are few. This is because a fully connected layer occupies most of the parameters, and hence, neurons develop co-dependency amongst each other during the training phase. It curbs the individual power of each neuron leading to over-fitting of training data and poor performance on the testing data [40]. In the proposed algorithm, the drop-out is achieved by setting a proportion of the outputs of some hidden neurons to zero. Then, they will not be considered in the forward propagation training process. The proportion here is set as 0.15 . However, in the testing phase, drop-out is switched off and all hidden neurons are included.
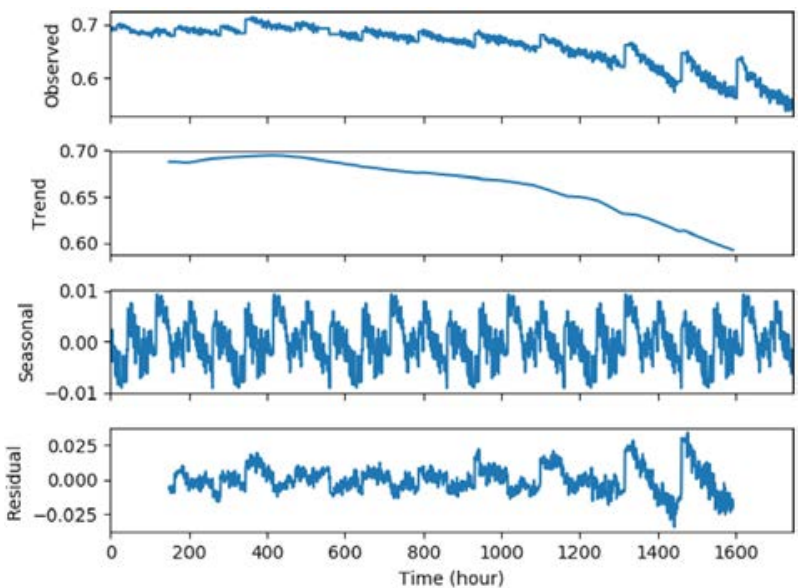

Fig. 4 - Decomposition result of $V_{\text {cell1 }}$. 
Table 2 - Key parameters of SDA model.

\begin{tabular}{llll} 
Structure parameters & & \multicolumn{2}{c}{ Training parameters } \\
\hline Input layer neurons & 4 & Transfer function & Sigmoid \\
Hidden layer 1 neurons & 16 & Batch size & 16 \\
Hidden layer 2 neurons & 8 & Epoch number & 20 \\
Output layer neurons & 1 & Sparsity criterion & 0.15 \\
\hline
\end{tabular}

\section{Performance metrics}

The performance of the proposed data-driven DT prognostics model is evaluated by two groups of evaluation metrics: accuracy and precision. Accuracy indicates quantitatively how accurate the predicted RULs are, while precision indicates the presence of uncertainty in the prediction results, which is an important index for the decision-making process. In this study, the accuracy is evaluated as relative accuracy (ReAcc) and by calculating the variance, the precision is evaluated based on the definition of the confidence interval limits. Both of the two metrics depends on the RUL calculation so that it is important to define the RUL calculation method. In this paper, direct RUL estimation method is used, which relies on the knowledge of the end-of-life, written as:

$R U L=t_{f}-t_{\lambda}$

where $t_{f}$ is the end-of-life of the system and $t_{\lambda}$ is the time instant of implementing prognostics. This RUL estimation method is commonly found in engine health prognostics and will be used in this study to justify the effectiveness of the proposed prognostics method [41]. Here, the end-of-life is set to 0.64 .

Then, the performance metrics are given below:

Relative accuracy

$\frac{\operatorname{ReAcC}=1-\left|\mathrm{RUL}^{*}-\widehat{\mathrm{RUL}}\right|}{\mathrm{RUL}^{*}}$

where RUL* is the real RUL and $\widehat{R U L}$ is the average output of the $N$ estimated RULs, calculated by

$\widehat{\mathrm{RUL}}=\frac{1}{N} \sum_{i=1}^{N} \widehat{\mathrm{RUL}} \widehat{\mathrm{i}}_{\mathrm{i}}$

Precision

Presicion $=\frac{\widehat{\mathrm{RUL}}^{\mathrm{CI}^{+}}-\widehat{\mathrm{RUL}}^{\mathrm{CI}^{-}}}{\mathrm{RUL}^{*}}$

$\widehat{\mathrm{RUL}}^{\mathrm{CI} \pm}=\widehat{\mathrm{RUL}} \pm \mathrm{z}^{*} \times \sqrt{\sigma^{2}}$

where $z^{*}$ is the critical standard Gaussian distribution value of a certain confidence level. For example, confidence level of $95 \%$ corresponds to a value of 1.96 , which is utilised in our case. $\sigma$ is the standard variation of the predicted RUL, which is calculated by,
$\sigma^{2}=\frac{1}{N} \sum_{i=1}^{N}\left(\widehat{R U L}_{i}-\widehat{R U L}\right)^{2}$

\section{Results evaluation}

The prognostics are performed every $50 \mathrm{~h}$ from the 100th hour until the 1100th hour during the online operation. A cross validation procedure is designed. The historical and test datasets are listed in Table 3.

The relative accuracy of the five tests versus different prediction starting instants are plotted with blue line in Fig. 5. For comparison, a prognostics method based on particle filtering (PF) approach using an exponential empirical model has been realized according to Jouin et al. [42]. The accuracy results are plotted with the orange line in Fig. 5. As it could be seen from the results, the accuracy of particle filtering method is lower than 0.9 and at certain instants, the prediction results are even worse than satisfied. This is due to the fact that there are not enough learning datasets and the prediction performance is highly dependent on the selection of model. On the contrary, the prediction performance of the proposed method rarely depends on the prediction horizon, i.e. even at the beginning of the test, the prediction accuracy is superior to that of PF prognostics method.

Fig. 6 shows the precision calculation results of the five tests at different prediction time. The precision of the prognostic results of the two methods have both showed an obvious increase as the prognostics horizon becomes smaller: this is because with more samples comes into the prognostics model, the predicted RULs will have a higher variation, while the precision of the proposed prognostics method shows a better performance than that of the PF prognostics method.

\section{Discussion}

The results of average accuracy of the proposed method and PF method with half and all measurement input are calculated in Table 4. The average accuracy of the proposed prognostics data-driven DT prognostics method has reached an average accuracy higher than 0.9 and near 0.95 , and a similar average accuracy higher than 0.9 even with only limited data input.

Based on the results, it has found that the proposed method benefits from the established DT structure so that the on-line prediction needs only small quantity of measurement data from the physical space, which is sufficient to update the digital model and output accurate RUL predictions. The realized deep transfer learning model based on SDA structure can

Table 3 - Training and testing datasets.

\begin{tabular}{lcc}
\hline Test No. & Historical datasets & Test dataset \\
\hline Test 1 & $\mathrm{U} 2, \mathrm{U} 3, \mathrm{U} 4, \mathrm{U} 5$ & $\mathrm{U} 1$ \\
Test 2 & $\mathrm{U} 1, \mathrm{U} 3, \mathrm{U} 4, \mathrm{U} 5$ & $\mathrm{U} 2$ \\
Test 3 & $\mathrm{U} 1, \mathrm{U} 2, \mathrm{U} 4, \mathrm{U} 5$ & $\mathrm{U} 3$ \\
Test 4 & $\mathrm{U} 1, \mathrm{U} 2, \mathrm{U} 3, \mathrm{U} 5$ & $\mathrm{U} 4$ \\
Test 5 & $\mathrm{U} 1, \mathrm{U} 2, \mathrm{U} 3, \mathrm{U} 4$ & $\mathrm{U} 5$ \\
\hline
\end{tabular}



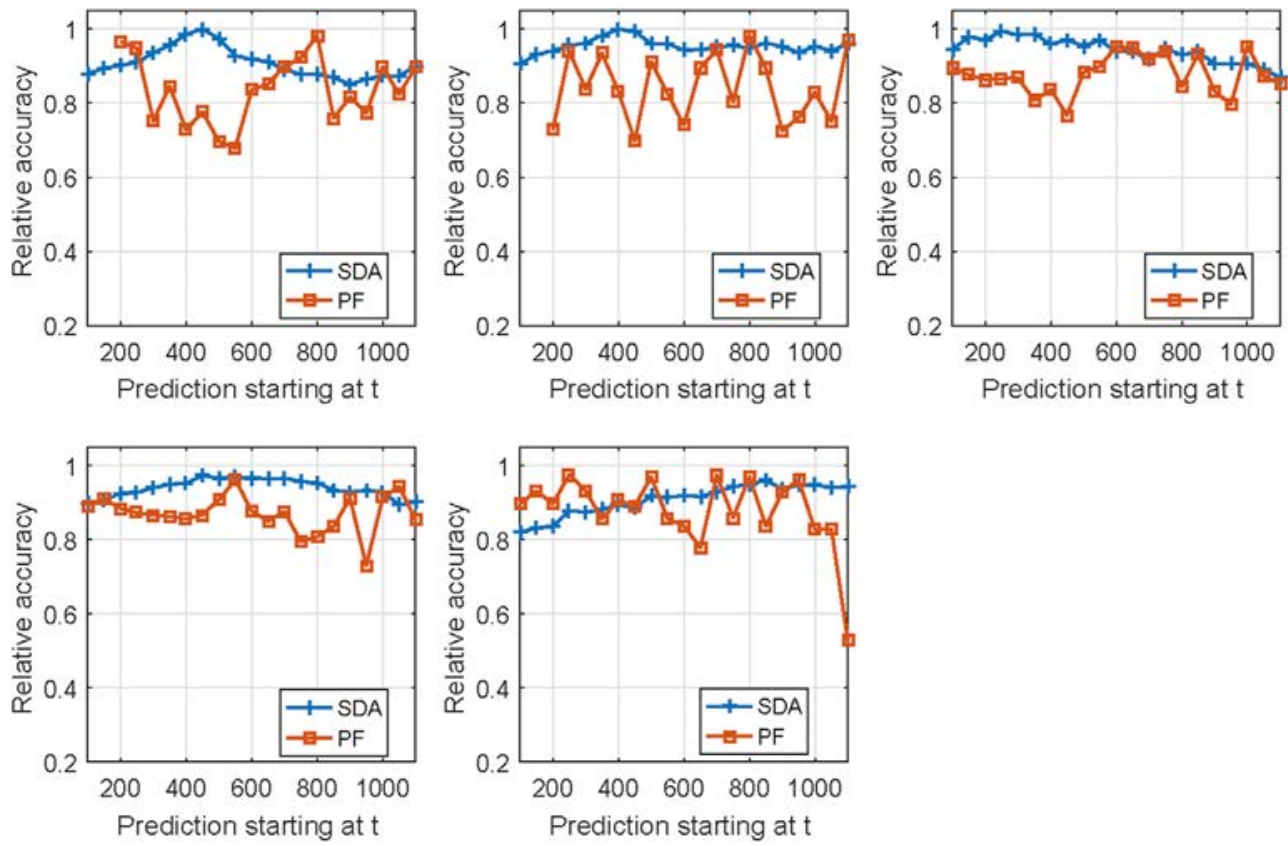

Fig. 5 - Relative accuracy comparison of the five tests using the proposed prognostics method and PF prognostics method.
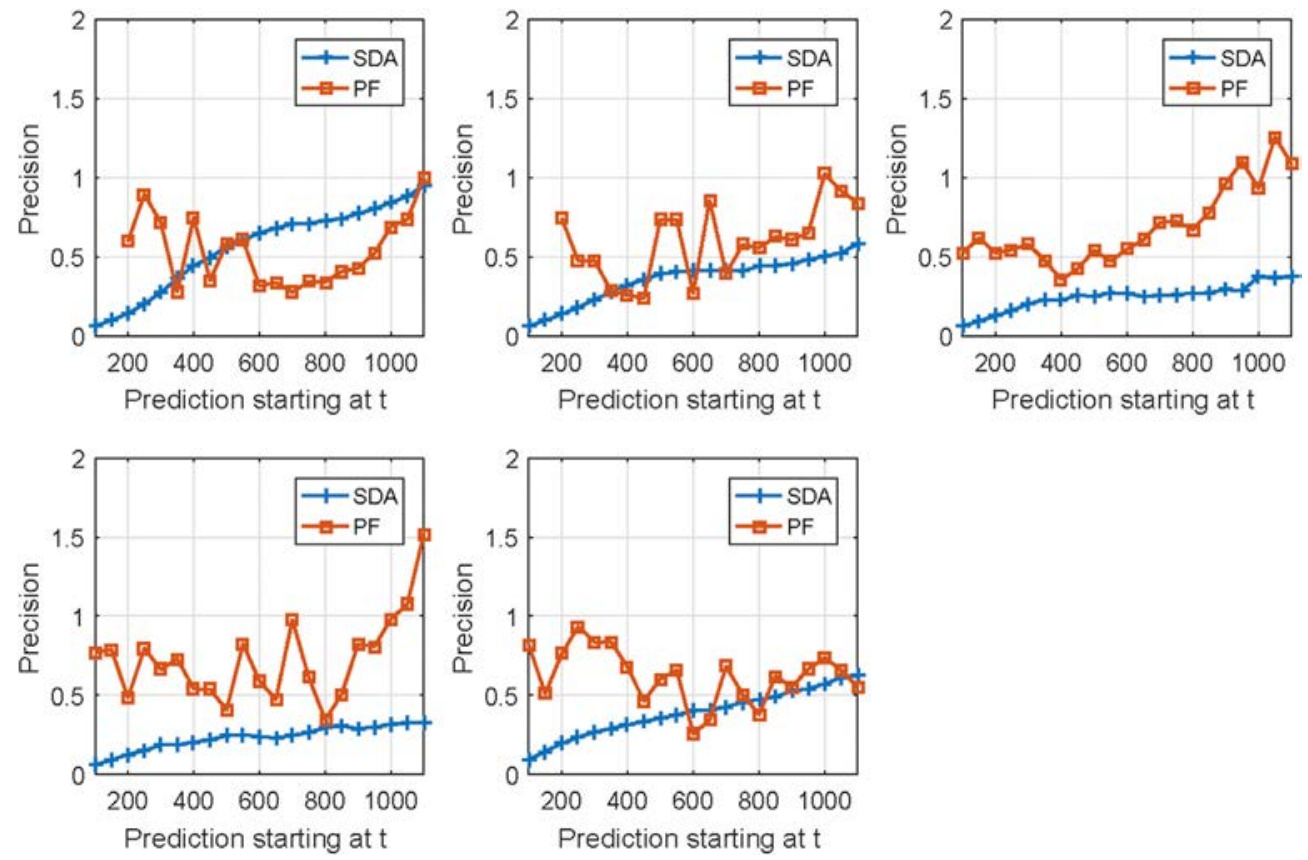

Fig. 6 - Precision comparison of the five tests using the proposed prognostics method and PF prognostics method.

physically present the degrading behaviour of the PEMFC, which can also be updated during real-time operation when similar fuel cells are observed. The trained SDA model is robust and can be used to predict RULs with limited input data, i.e. from the very beginning of the fuel cell life. Besides, the physics model-free nature of the proposed method avoids the dependence on selecting prediction models, which makes it a competitive prognostics approach in PEMFC applications.

However, to scale up industrial applications of the developed data-driven DT prognostics method, some challenges are facing: (1) the digital side of the DT is expected to be constructed with more complicated measurements under

Table 4 - Comparison of the average relative accuracy.

Test 1 Test 2 Test 3 Test 4 Test 5

$\begin{array}{llllll}\text { Data-driven DT (first 500 h) } & 0.971 & 0.940 & 0.873 & 0.937 & 0.960\end{array}$

$\begin{array}{llllll}\text { Data-driven DT (all) } & 0.908 & 0.954 & 0.943 & 0.940 & 0.908\end{array}$

$\begin{array}{llllll}\text { PF (first } 500 \mathrm{~h} \text { ) } & 0.803 & 0.827 & 0.851 & 0.880 & 0.917\end{array}$

$\begin{array}{llllll}\text { PF (all) } & 0.830 & 0.846 & 0.879 & 0.872 & 0.900\end{array}$


different operation conditions; (2) cost-effectiveness of the proposed method, i.e. the computation burden of the algorithm, should be considered regarding the capability of the industrial server.

\section{Conclusion}

This paper has proposed a date-driven DT prognostics method to predict the RULs for PEMFC applications. As PEMFC is usually operated in complex systems, its health state needs to be monitored and predicted accurately so that adequate actions could be conducted to ensure the integrity and reliability of the system. The proposed data-driven DT prognostics method is able to update the PEMFC degradation model on the digital side with the online measurements from the physical side, which improves the prognostics performance considering the changing operating conditions and intercell differences. The degradation model on the digital side of DT is built using an SDA, which models the RUL of the PEMFC directly from the input stack voltage. It is updated by connecting to the physical side of DT where online measurements are collected. Results show that the proposed prognostics method can effectively predict the RUL of the target PEMFC with an average accuracy higher than 0.9 and the predicted results are less affected even with limited measurement data. For the perspectives, a decision-making module is expected to be added to provide following predictive maintenance actions.

\section{Declaration of competing interest}

The authors declare that they have no known competing financial interests or personal relationships that could have appeared to influence the work reported in this paper.

\section{Acknowledgment}

The authors would like to thank the project PHyTie (grant number 2016Y-04574) funded by the French region of Bourgogne Franche-Comté for the support. This work has also been supported by the EIPHI Graduate School (contract ANR17-EURE-0002) of the Region Bourgogne-Franche-Comté, France.

\section{R EFER E N C E S}

[1] Rivarolo Massimo, Rattazzi Diego, Lamberti Thomas, Magistri Loredana. Clean energy production by pem fuel cells on tourist ships: a time-dependent analysis. Int J Hydrogen Energy 2020;45(47):25747-57.

[2] Haseli Yousef. Maximum conversion efficiency of hydrogen fuel cells. Int J Hydrogen Energy 2018;43(18):9015-21.

[3] Brooks Kriston P, Sprik Samuel J, Tamburello David A, Thornton Matthew J. Design tool for estimating metal hydride storage system characteristics for light-duty hydrogen fuel cell vehicles. Int J Hydrogen Energy 2020;45(46):24917-27.
[4] Yue Meiling, Jemei Samir, Gouriveau Rafael, Zerhouni Noureddine. Review on health-conscious energy management strategies for fuel cell hybrid electric vehicles: degradation models and strategies. Int J Hydrogen Energy 2019;44(13):6844-61.

[5] Gharibi Mahdi, Askarzadeh Alireza. Size and power exchange optimization of a grid-connected diesel generatorphotovoltaic-fuel cell hybrid energy system considering reliability, cost and renewability. Int J Hydrogen Energy 2019;44(47):25428-41.

[6] Vichard Loïc, Harel Fabien, Ravey Alexandre, Pascal Venet, Hissel Daniel. Degradation prediction of pem fuel cell based on artificial intelligence. Int J Hydrogen Energy 2020;45(29):14953-63.

[7] Omri N, Masry AZ, Mairot N, Giampiccolo S, Zerhouni N. Industrial data management strategy towards an smeoriented phm. J Manuf Syst 2020;56(23-36).

[8] Liu Hao, Chen Jian, Hou Ming, Shao Zhigang, Su Hongye. Databased short-term prognostics for proton exchange membrane fuel cells. Int J Hydrogen Energy 2017;42(32):20791-808.

[9] Chen Kui, Laghrouche Salah, Abdesslem Djerdir. Fuel cell health prognosis using unscented kalman filter: postal fuel cell electric vehicles case study. Int J Hydrogen Energy 2019;44(3):1930-9.

[10] Morando Simon, Jemei S, Gouriveau Rafael, Zerhouni Noureddine, Hissel Daniel. Fuel cells prognostics using echo state network. In: IECON proceedings (industrial electronics conference); 2013. p. 1632-7. 11.

[11] Silva RE, Gouriveau R, Jemei S, Hissel D, Boulon L, Agbossou K, Yousfi-Steiner N. Proton exchange membrane fuel cell degradation prediction based on adaptive neurofuzzy inference systems. Int J Hydrogen Energy 07 2014;39:11128-44.

[12] Javed Kamran, Gouriveau Rafael, Zerhouni Noureddine, Hissel Daniel. Improving accuracy of long-term prognostics of pemfc stack to estimate remaining useful life. In: 2015 IEEE international conference on industrial technology (ICIT). IEEE; March 2015. p. 1047-52.

[13] Bouzidi Zohra, Terrissa Labib Sadek, Zerhouni Noureddine, Ayad Soheyb. An efficient cloud prognostic approach for aircraft engines fleet trending. Int J Comput Appl 2020;42(5):514-29.

[14] Benaggoune K, Mouss LH, Abdessemed AA, Bensakhria M. Holonic agent-based approach for system-level remaining useful life estimation with stochastic dependence. Int J Comput Integrated Manuf 2020:1-16.

[15] Ayoub Benayache, Bilami Azeddine, Barkat Sami, Lorenz Pascal, Taleb Hafnaoui. Msm: a microservice middleware for smart wsn-based iot application. J Netw Comput Appl 2019;144:138-54.

[16] Lu Yuqian, Liu Chao, Kevin I, Wang Kai, Huang Huiyue, Xu Xun. Digital Twin-driven smart manufacturing: connotation, reference model, applications and research issues. Robot Comput Integrated Manuf 2020;61(July 2019):101837.

[17] Boschert Stefan, Rosen Roland. Digital twin-the simulation aspect. Mechatronic futures. Springer; 2016. p. 59-74.

[18] Tuegel Eric J, Ingraffea Anthony R, Eason Thomas G, Spottswood S Michael. Reengineering aircraft structural life prediction using a digital twin. International Journal of Aerospace Engineering 2011;2011.

[19] Tao Fei, Zhang Meng, Liu Yushan, Nee AYC. Digital twin driven prognostics and health management for complex equipment. CIRP Annals 2018;67(1):169-72.

[20] Xie Jiacheng, Wang Xuewen, Yang Zhaojian, Hao Shangqing. Virtual monitoring method for hydraulic supports based on digital twin theory. Min Technol 2019;128(2):77-87.

[21] Zakrajsek Andrew J, Mall Shankar. The development and use of a digital twin model for tire touchdown health monitoring. 
In: 58th AIAA/ASCE/AHS/ASC structures, structural dynamics, and materials conference; 2017. 0863.

[22] Xu Yan, Sun Yanming, Liu Xiaolong, Zheng Yonghua. A digital-twin-assisted fault diagnosis using deep transfer learning, vol. 7. IEEE Access; 2019. p. 19990-9.

[23] Wang Jinjiang, Ye Lunkuan, Gao Robert X, Chen Li, Zhang Laibin. Digital twin for rotating machinery fault diagnosis in smart manufacturing. Int J Prod Res 2019;57(12):3920-34.

[24] Francisco Abigail, Mohammadi Neda, Taylor John E. Smart city digital twin-enabled energy management: toward realtime urban building energy benchmarking. J Manag Eng 2020;36(2). 04019045.

[25] O'Dwyer Edward, Pan Indranil, Charlesworth Richard, Butler Sarah, Shah Nilay. Integration of an energy management tool and digital twin for coordination and control of multi-vector smart energy systems. Sustainable Cities and Society 2020;62:102412.

[26] Karanjkar Neha, Joglekar Ashish, Mohanty Sampad, Prabhu Venkatesh, Raghunath D, Sundaresan Rajesh. Digital twin for energy optimization in an smt-pcb assembly line. In: 2018 IEEE international conference on internet of things and intelligence system (IOTAIS). IEEE; 2018. p. 85-9.

[27] Snijders Ron, Pileggi Paolo, Broekhuijsen Jeroen, Verriet Jacques, Marco Wiering, Kok Koen. Machine learning for digital twins to predict responsiveness of cyber-physical energy systems. In: 2020 8th workshop on modeling and simulation of cyber-physical energy systems. IEEE; 2020. p. 1-6.

[28] Wang Bowen, Zhang Guobin, Wang Huizhi, Jin Xuan, Jiao Kui. Multi-physics-resolved digital twin of proton exchange membrane fuel cells with a data-driven surrogate model. Energy and AI 2020;1:100004.

[29] Zhuang Cunbo, Liu Jianhua, Xiong Hui. Digital twin-based smart production management and control framework for the complex product assembly shop-floor. Int J Adv Manuf Technol 2018;96(1-4):1149-63.

[30] Tao Fei, Cheng Jiangfeng, Qi Qinglin, Zhang Meng, Zhang He, Sui Fangyuan. Digital twin-driven product design, manufacturing and service with big data. Int J Adv Manuf Technol 2018;94(9-12):3563-76.

[31] Min Qingfei, Lu Yangguang, Liu Zhiyong, Su Chao, Wang Bo. Machine learning based digital twin framework for production optimization in petrochemical industry. Int J Inf Manag 2019;49:502-19.

[32] Han Jaesu, Han Jaeyoung, Yu Sangseok. Experimental analysis of performance degradation of 3-cell pemfc stack under dynamic load cycle. Int J Hydrogen Energy 2020;45(23):13045-54.

[33] Zhang Xuexia, Zhou Jingzhe, Chen Weirong. Data-driven fault diagnosis for pemfc systems of hybrid tram based on deep learning. Int J Hydrogen Energy 2020;45(24):13483-95.

[34] Vincent Pascal, Larochelle Hugo, Bengio Yoshua, Manzagol Pierre-Antoine. Extracting and composing robust features with denoising autoencoders. In: Proceedings of the 25th international conference on machine learning, ICML '08. New York, NY, USA: Association for Computing Machinery; 2008. p. 1096-103.

[35] Bengio Yoshua, Pascal Lamblin, Popovici Dan, Larochelle Hugo. Greedy layer-wise training of deep networks. In: Schölkopf B, Platt JC, Hoffman T, editors. Advances in neural information processing systems 19. MIT Press; 2007. p. 153-60.

[36] Quoc V Le, Google Brain, Google Inc. A tutorial on deep learning part 2: autoencoders, convolutional neural networks and recurrent neural networks. 2015.

[37] Morando Simon, Jemei Samir, Hissel Daniel, Gouriveau Rafael, Zerhouni Noureddine. Proton exchange membrane fuel cell ageing forecasting algorithm based on echo state network. Int J Hydrogen Energy 2017;42(2):1472-80.

[38] Jouin Marine, Gouriveau Rafael, Hissel Daniel, Péra MarieCécile, Zerhouni Noureddine. Degradations analysis and aging modeling for health assessment and prognostics of pemfc. Reliab Eng Syst Saf 2016;148:78-95.

[39] Jouin Marine, Gouriveau Rafael, Hissel Daniel, MarionPéra Marie-Cécile, Zerhouni Noureddine. Degradations analysis and aging modeling for health assessment and prognostics of pemfc. Reliab Eng Syst Saf 2016;148:78-95.

[40] Xiang Li, Ding Qian, Sun Jian-Qiao. Remaining useful life estimation in prognostics using deep convolution neural networks. Reliab Eng Syst Saf 2018;172:1-11.

[41] Lu Chen, Wang Zhen-Ya, Qin Wei-Li, Ma Jian. Fault diagnosis of rotary machinery components using a stacked denoising autoencoder-based health state identification. Signal Process 2016;130. 07.

[42] Jouin Marine, Gouriveau Rafael, Hissel Daniel, Zerhouni Noureddine, Marion-Péra Marie-Cécile. Prognostics of proton exchange membrane fuel cell stack in a particle filtering framework including characterization disturbances and voltage recovery. In: 2014 international conference on prognostics and health management, PHM; 2014. 062014. 